\title{
An Innovative Complex of Benzene-Poly-Carboxylic Acid and Molybdenum, for Prevention and Treatment of Radiation Dermatitis
}

\section{Fuad Fares $^{1 *}$, Basem Fares ${ }^{1}$, Naiel Azzam ${ }^{1}$, Munir Nashashibi ${ }^{2}$, Alex Nevelsky ${ }^{3}$, Stig Larsen ${ }^{4}$ and Steen Lindkær-Jensen ${ }^{5}$}

${ }^{1}$ Department of Human Biology, Faculty of Natural Sciences, University of Haifa, Haifa, Israel

${ }^{2}$ Department of Pathology, Carmel Medical Center, Haifa, Israel

${ }^{3}$ Department of Oncology, Rambam Health Care Center, Haifa, Israel

${ }^{4}$ Department of Controlled Clinical Trials and Biostatistics, University of Oslo, Oslo, Norway

${ }^{5}$ Department of Surgery and Cancer, Imperial College, Hammersmith Hospital, DuCane Road, W12 oNN London, United Kingdom

\begin{abstract}
Radiation dermatitis occurs in up to 95 percent of patients receiving radiotherapy. The aim of this study was to evaluate whether topical application of a new Benzene-Poly-Carboxylic Acid and Molybdenum Complex (BP-C2) prophylactically could protect mice skin from irradiation-induced dermatitis or treat irradiation-induced skin dermatitis, when it already has occurred. The right posterior leg of male BALB/cfC3H mice was exposed to radiation dose of 30 Gy. For prevention studies, animals were treated with BP-C2 just prior to irradiation and three times a week for 5 weeks post-radiation. For treatment studies, animals were treated with PB-C2 three times a week for five weeks when skin injury was appeared following irradiation exposure. Animals were sacrificed and skin damage was assessed using a non-linear semi-quantitative scale, as well as histological studies. Acute skin dermatitis was observed in all control animals $(n=8)$ following $30 \mathrm{~Gy}$ irradiation in form of edema and ulceration grade 4 . No signs of skin dermatitis were observed in the irradiated area of animals $(n=8)$ treated with BP-C2 prior to irradiation exposure. Moreover, treatment of injured animals with BP-C2 three times a week resulted in recovery of the skin and no erythema or ulceration was hereafter observed. BP-C2 represents a potentially promising agent for prevention and treatment of irradiation-induced skin dermatitis.
\end{abstract}

Keywords: BP-C2; Skin dermatitis; Electron beam irradiation; Prevention; Treatment

Abbreviations: BP-C2: Benzene-Poly-Carboxylic Acid and Molybdenum Complex; IVCs: Individually ventilated cages; SPF: In a specific pathogen free; H\&E: Hematoxylin and Eosin; TGF- $\beta$ : Tumor Growth Factor; IMRT: Introduction to the Intensity-modulated Radiation Therapy; 3D-CRT: Conformal Radiation Therapy

\section{Introduction}

Irradiation dermatitis occurs in up to 95 percent of cancer patients receiving radiotherapy, especially in patients with breast cancer, head and neck cancer, lung cancer, or sarcoma due to a higher radiation dose to the skin [1-7]. In most cases, the skin reaction is mild or moderate. Approximately 20 to 25 percent of patients experience moist desquamation and ulceration [8-10]. Irradiation-induced dermatitis has a profound impact on the quality of a patient's life, due to pain, discomfort and premature interruption of radiation therapy, resulting in inadequate cancer treatment. Irradiation-induced fibrosis may cause both cosmetic and functional impairment, which can lead to death or significant deterioration in quality of life. Different areas of the body have different sensitivities to radiation dermatitis. The most sensitive regions of the body are the anterior of the neck, extremities, chest, abdomen, and face [3].

The National Cancer Institute (NCI) in United States has developed a 4 stage criteria for classification of acute radiation dermatitis: a) Grade I - Faint erythema or desquamation, b) grade II - Moderate to brisk erythema or patchy, moist desquamation confined to skin folds and creases. Moderate swelling, c) grade III - Confluent, moist desquamation greater than $1.5 \mathrm{~cm}$ diameter, which is not confined to the skin folds. Pitting edema (severe swelling), and d) grade IV - Skin necrosis or ulceration of full thickness dermis (middle layer of skin) $[2,11,12]$.

It is well known especially from the Russian literature that a complex of organic compounds with variable-valence metals, especially platinum and manganese, and molybdenum has radio-protective properties [13]. BP-C2 is a complex of BP-Cx-1 and molybdenum. BP-Cx-1 is a complex of benzene-polycarboxylic acids and it is known for its detoxifying free-radical, anti-oxidant and anticancer activities $[14,15]$. Intragastric application of BP-C2 was performed in CBA mice and Wistar rats. Repeated intragastric administration of BP-C2 promoted radioprotection against radiation doses in the median lethal dose range. The optimal radio-protective doses were $81.0 \mathrm{mg} / \mathrm{kg} \mathrm{BW}$ for mice and $93.7 \mathrm{mg} / \mathrm{kg}$ BW for rats [13]. No topical application of $\mathrm{BP}-\mathrm{C} 2$ was used. The conclusion was that the use of BP-C2 promotes a favorable course and increases the likelihood of a favorable outcome of acute irradiation injury. The available Russian literature suggest promising area of research, namely creating effective anti-radiation pharmacological means for medical protection with characteristics that meet the current high standards for effectiveness and convenience are complexes of organic compounds and variable-valence metals [13]. On the base of these facts, the present study aims to test the potential of BP$\mathrm{C} 2$, an innovative molecule, on prevention and treatment of radiationinduced skin dermatitis using a mice model.

\section{Experimental Methods}

\section{Chemical and biological reagents}

BP-C2 (Figure 1) was kindly supplied by Meabco Oncology

*Corresponding author: Fuad Fares, Department of Human Biology, Faculty of Natural Sciences, University of Haifa, 31095, Israel, Tel: 972-4-8288781; Fax: 972 4-8288763; E-mail: ffares@sci.haifa.ac.ilc

Received September 28, 2015; Accepted October 12, 2015; Published October 15,2015

Citation: Fares F, Fares B, Azzam N, Nashashibi M, Nevelsky A, et al. (2015) An Innovative Complex of Benzene-Poly-Carboxylic Acid and Molybdenum for Prevention and Treatment of Radiation Dermatitis. Med chem 5: 447-451. doi:10.4172/2161-0444.1000299

Copyright: $\odot 2015$ Fares F, et al. This is an open-access article distributed under the terms of the Creative Commons Attribution License, which permits unrestricted use, distribution, and reproduction in any medium, provided the original author and source are credited. 


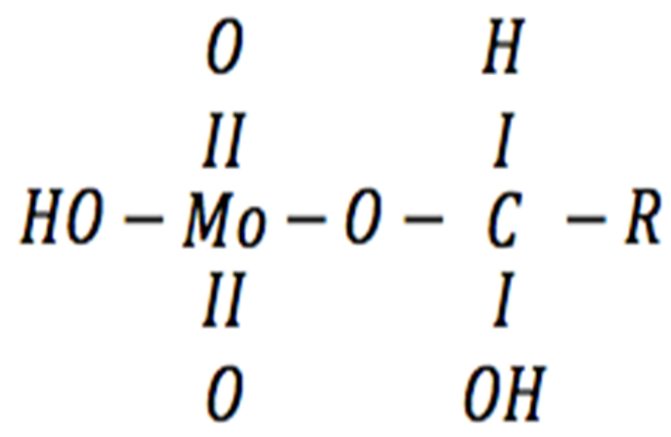

Figure 1: Chemical structure of BP-C2 analog. R- fragment of the ligand with molecular weight of $475 \mathrm{Da}$.

A/S, Copenhagen, Denmark. The synthesis of BP-C2 solution was done in a single step starting from hydrolyzed humic acid (BP-Cx-1 ligand) and ammonium molybdate tetrahydrate (Brutto formula: $\left.\left(\mathrm{NH}_{4}\right)_{6} \mathrm{Mo}_{7} \mathrm{O}_{24}{ }^{*} 4 \mathrm{H}_{2} \mathrm{O}, \mathrm{MM} 1235 \mathrm{~g} / \mathrm{mol}\right)$ in water in presence of $10 \%$ aq ammonia under ultrasound treatment at $22.0 \mathrm{kHz}$ for ten minutes. The ligand, BP-Cx-1, is a polymer of benzene poly-carboxylic acids, obtained by alkaline oxidation from hydrolyzed lignin. All other chemicals were purchased from Sigma (Saint Louis, MO, USA) or other local sources. $\mathrm{LD}_{50}$ of BP-C2 is $8000 \mathrm{mg} / \mathrm{kg}$. Composition of $1 \mathrm{ml}$ of the trial medication BP-C2 is $1.5 \mathrm{mg}$ of the amount of molybdenumammonium salts of benzene -poly-carbonic acids, including $0.2 \mathrm{mg}$ of Molybdenum.

\section{Animals}

Male BALB/cfC3H mice, 5 weeks old; $18-20$ g body weights were purchased from Harlan Laboratories Jerusalem, Israel. Experiments were performed with 8 mice in each group. The animal protocols used in this work were evaluated and approved by the Ethics Commission on Animal Use (CEUA) of the Technion-Israel Institute of Technology. They are in accordance with FELASA guidelines and the National law for Laboratory Animal Experimentation (Law no. 18.611).

All mice were held in individually ventilated cages (IVCs) in a specific pathogen free (SPF) barrier unit with 5 mice in each cage. There was a constant room temperature of $20^{\circ} \mathrm{C}$ and a mean humidity of $55 \%$. The animals received 2018 extruded rodent diet (Harlan Laboratories) and sterile acidified water (ad libitum) from time of arrival and throughout the study.

\section{Radiation treatment}

$6 \mathrm{MeV}$ electron beam from a medical linear accelerator (ELEKTA $\mathrm{AB}$, Stockholm, Sweden) was used for irradiation. In order to facilitate local irradiation, a special cut-out insert with a circular $2 \mathrm{~cm}$ diameter aperture was prepared. A $0.5 \mathrm{~cm}$ tissue equivalent bolus was used to bring the maximal dose to the skin surface. The radiation output in the center of the aperture was measured with an ionization chamber. Mice were shaved in the leg side and anesthetized with an intraperitoneal injection of ketamine $(100 \mathrm{mg} / \mathrm{kg})$ and xylazine $(8 \mathrm{mg} / \mathrm{kg})$. After 10 minutes, the right posterior leg of the animals was irradiated with a total dose of $30 \mathrm{~Gy}$ as described before [16]. $30 \mathrm{~Gy}$ was given in row of 5 times $6 \mathrm{GY} /$ time. Histological findings of radiation-induced dermatitis were determined as described previously [17].

\section{Treatment with BP-C2}

For prevention studies, 5 weeks old animals were divided into two groups; control and treated. Prior to irradiation exposure the treated group, animals were treated with BP-C2 and 3 times a week after irradiation for 10 weeks (Figure 2). For treatment studies, animals were irradiated with $30 \mathrm{~Gy}$, and after 5 weeks when skin injury was observed animals were treated 3 times a week with BP-C2 for 5 weeks (Figure 3). At the end of the experiments, animals were sacrificed and uniformed taken skin biopsies were prepared for histopathological studies. The vehicle is physiologic saline and the control group consists of animals sprayed with physiologic saline, i.e., the saline treated group is a proper control group treated with the vehicle.

\section{Skin effects}

Following irradiation mice were thoroughly inspected several times per week and the quality of the skin was recorded by an independent pathologist. For monitoring the radiation dermatitis, photographs of the animals were taken twice a week and the skin injury score was assessed as follows: (0) normal, (1) erythema, (2) dry desquamation, (3) wet desquamation, and (4) ulceration. Skin damage was assessed using a non-linear semi-quantitative scale and by conventional histopathology. Skin damage was measured several times per week for 10 weeks.

\section{Hematoxylin and eosin staining protocol}

All histopathological procures were carried out at the Department of Pathology, Carmel Medical Center, Haifa, Israel, Paraffin sections were de-waxed in xylene and rehydrated through a series of alcohol

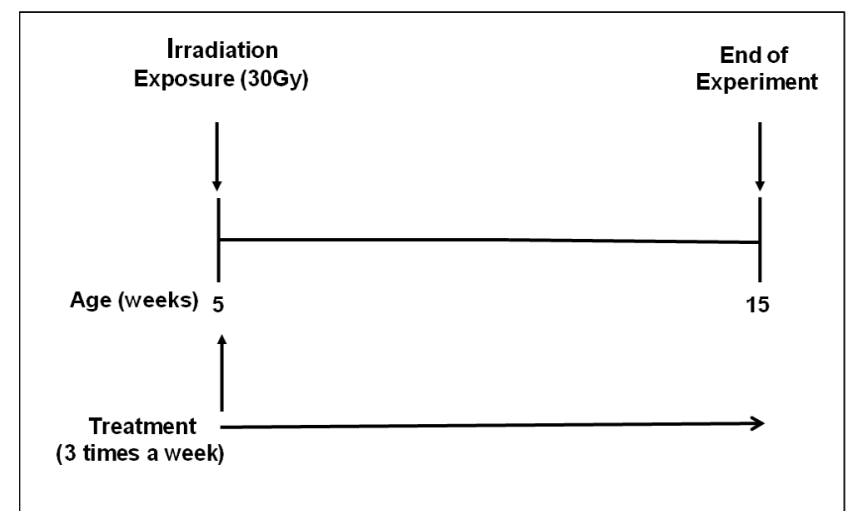

Figure 2: A schematic, prophylactic treatment regimen with BP-C2 of acute skin dermatitis in mice. For prevention studies, animals were treated with $\mathrm{BP}-\mathrm{C} 2$ prior to irradiation exposure. After irradiation exposure to $30 \mathrm{~Gy}$, animals were treated 3 times a week with BP-C2 for 10 weeks.

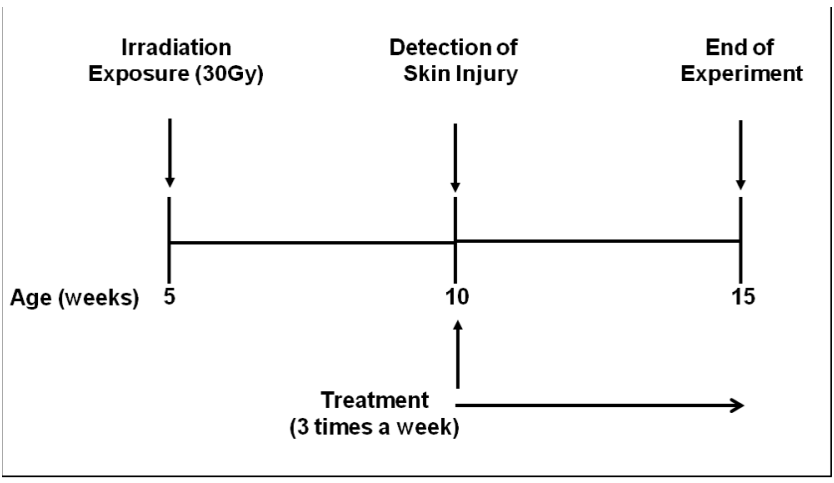

Figure 3: A schematic treatment regimen with BP-C2 after occurrence of acute skin dermatitis. Animals were exposed to irradiation, $30 \mathrm{~Gy}$, and after 5 weeks when skin injury was observed, animals were treated 3 times a week with BP-C2 for 5 weeks. 
to water using a Leica Autostainer XL ST5010. Once they were rehydrated, the slides were immersed in Gill's 2 Hematoxylin (ThermoFischer 6765007, Thermo-Fischer Scientific, Bishop Meadow Fischer, Loughborough, UK) for 1 minute, rinsed in running water for 1 minute, dipped in alkaline water, and then rinsed before being immersed in Eosin (Thermo-Fishern 6765009) for 1 minute. Sections were then rinsed in water, dehydrated and cleared in xylene using the Leica Autostainer XL before being permanently mounted.

\section{Statistics}

Contingency table analysis was used for statistical analysis. The main variable in this study was recorded on an ordinal 4-point fixed scale. The recommended model for performance of analysis of such variables is Contingency Table Analysis as performed. Analysis of Variance (ANOVA) demands continuously and normal distribution $\mathrm{N}$ $(\mu ; \sigma)$ and even Dunn's model of ANOVA on Ranks will lose power with an outcome space including max 4 outcome. The tie configuration will destroy the power of a model and will obviously weaker compare to Contingency Table Analysis. $\mathrm{P}<0.05$ was considered significant.

\section{Results}

\section{Prophylactic studies}

In order to test the efficacy of $\mathrm{BP}-\mathrm{C} 2$ on prevention of radiation induced skin injury, animals were treated with BP-C2 before radiation exposure and 3 times a week for more 10 weeks (Figure 2). The results indicated that acute skin reactions were observed in all control animals (8 animals/group) following $30 \mathrm{~Gy}$ of irradiation. The irradiated areas demonstrated dermatitis edema and ulceration grade IV. Interestingly, no skin dermatitis was observed in the irradiated area of animals the prophylactically treated with $\mathrm{BP}-\mathrm{C} 2,(\mathrm{p}<0.01)$ between the two groups. Representative macroscopic comparison of untreated control and treated animals with BP-C2 before and 3 times a week after irradiation are shown in Figure 4.

\section{Treatment studies}

In order to test the efficacy of BP-C2 on the treatment of radiation induced skin injury, animals that were injured by radiation exposure were treated 3 times a week for 5 weeks (Figure 3 ). The results indicate that all control animals ( 8 animals/group) were developed breakdown of the epidermal barrier and ulcerations grade IV after exposure to $30 \mathrm{~Gy}$. Treatment of injured animals (8 animals/group) with BPC2, 3 times a week for 5 weeks resulted in recovery of the skin and
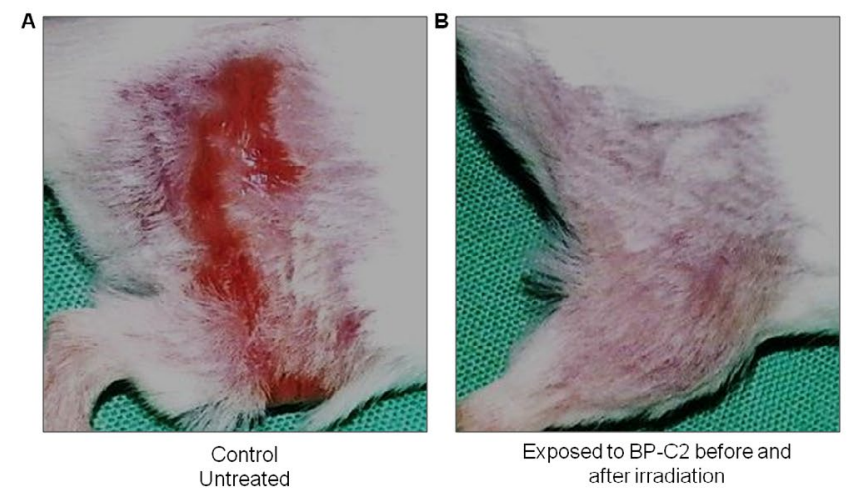

Figure 4: Macroscopic comparison of radiation dermatitis in representative mice. Animals were exposed to irradiation, $30 \mathrm{~Gy}$, without (A) or with BP$\mathrm{C} 2$ treatment $(\mathrm{B})$. Animals were treated with $\mathrm{BP}-\mathrm{C} 2$ prior to irradiation and 3 times a week. After 10 weeks animals were sacrificed and skin biopsies were collected. no erythema or ulceration were observed $(\mathrm{p}<0.001)$. Representative macroscopic comparison of control and treated animals are shown in Figure 5. The results indicated that BP-C2 treatment recovered the skin injury induced by irradiation.

\section{Histopathological studies}

For histological evaluation of skin biopsies from control and treated animals, skin biopsies were fixed in formalin and paraffin blocks were prepared. Four micron sections were prepared, stained with hematoxylin and eosin ( $\mathrm{H} \& \mathrm{E})$ and examined as described under "Methods and Materials". Ten weeks post-irradiation skin tissue shows severe irradiation injury results with complete loss of the epidermis and persistent fibrin exudates, edema and ulceration covered by fibrininflammatory debris grade IV (Figure 6A). However, in skin biopsied from treated animals with BP-C2 prior to irradiation exposure and 3 times a week for 10 weeks, a mild injury in the hair roots was observed and scattered melanin granules were present within the hair and hair roots (Figure 6B). Five weeks post irradiation skin biopsies from representative animals show loss of epidermis, edema and ulceration covered by fibrin-inflammatory debris grade IV (Figure 6C). On the other hand, treatment of the injured animals with BP-C2, 3 times a week for 5 weeks, showed recovery of skin tissue with atrophy of epidermal layers, dermal structures were presented without ulceration. In addition, hair follicles were present with scattered melanin granules however, very mild edema and fibrosis was detected in the superficial dermis with mild chronic inflammation (Figure 6D).

\section{Discussion}

The present study has shown that topical application of BP-C2 protects against irradiation-induced dermatitis in mice whether BPC2 is used prophylactically or as a treatment after skin dermatitis has occurred. We have demonstrated that when BP-C2 is used prophylactically no signs of skin dermatitis can be observed. When skin ulcers have occurred after irradiation they disappear completely after topical application of BP-C2; this is confirmed macroscopically as well as by histopathological examinations. Histopathological examination demonstrated almost normal skin biopsies after treatment with BPC2 without ulceration but with mild edema, mild fibrosis, and mild chronic inflammation. The effect on skin ulcers together with nontoxicity or side effects, makes BP-C2 a promising drug for treatment of radiotherapy induced skin dermatitis. Skin dermatitis is not only seen after radiotherapy for cancer but also as a complication to post-radiation exposure from accidents or weapons, such as "dirty bomb" [6]. The
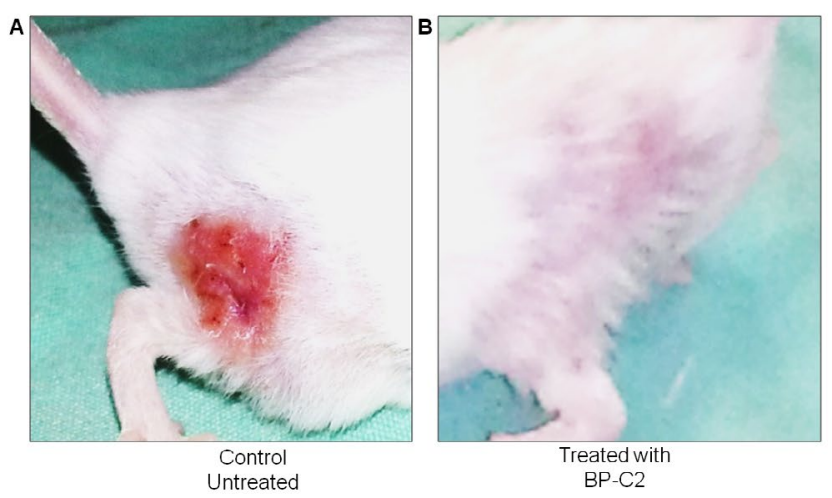

Figure 5: Macroscopic comparison of radiation dermatitis in representative mice without (A) or with BP-C2 treatment (B) after exposure to $30 \mathrm{~Gy}$. Animals were exposed to irradiation, $30 \mathrm{~Gy}$, and after 5 weeks when skin injury was observed, animals were treated 3 times a week with BP-C2 for 5 weeks. 

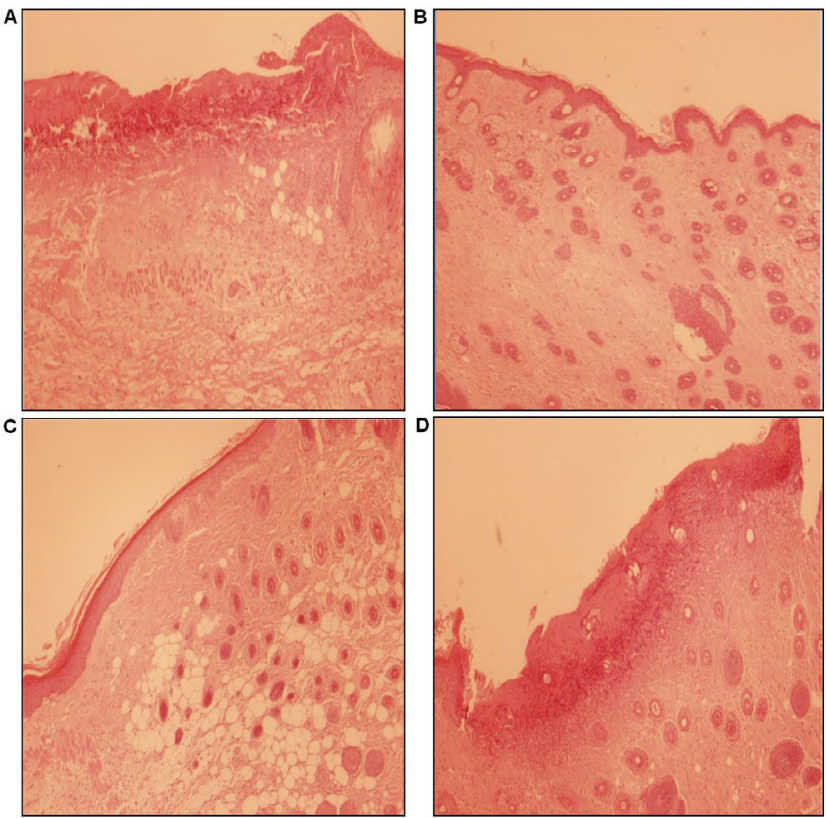

Figure 6: Histopathological evaluation of skin biopsies. Paraffin blocks were prepared and four micron sections were stained with hematoxylin and eosin (H\&E). For prevention studies, animals were divided into two groups; control (A) and treated (B). Prior to irradiation exposure the treated group were treated with BP-C2 followed by treatment 3 times a week after irradiation for 10 weeks (B). For treatment studies, animals were irradiated with $30 \mathrm{~Gy}$, and after 5 weeks when skin injury was observed animals were treated 3 times a week with BP-C2 for 5 weeks (D) and compare to untreated animals (C). In the superficial dermis of untreated animals ( $A$ and $C$ ), edema, fibrosis and chronic inflammation were detected. On the other hand, treatment with BP-C2 irradiation exposure showed intact epidermal barrier with very little edema, very mild fibrosis and very mild chronic inflammation without ulceration (B and $\mathrm{C})$.

irradiation-induced toxicity such as skin dermatitis can result in poor outcome due to interrupted or limited radiotherapy adversely affecting quality of life [18]. Therefore, it is of utmost importance to develop nontoxic anti-skin dermatitis treatment to be used during radiotherapy without impairing the antitumor efficacy of the irradiation.

The Danish pharmaceutical company Meabco A/S has recently developed a new substance based on the BP-C2, a novel complex of benzene-poly-carboxylic acids with a very low concentration of molybdenum, which from a theoretical point of view should have a strong impact on irradiation-induced dermatitis and on healing of irradiated-induced skin dermatitis. So far we have shown in vitro and in in vivo experiments that BP-C2 is a strong modulator of the innate immune system and that it corrects hematological abnormalities such as anemia, thrombocytopenia and neutropenia often seen in patients with cancer [19]. Other mechanisms of action (MoA) involved could be DNA damage and improve DNA repair after irradiation, the presence of the so-called membranotropic effect and effects on various genes and cytokines [20].

Radiotherapy induced skin dermatitis exists in an acute and a chronic form $[21,22]$. In this study we use a high single radiation dose of $30 \mathrm{~Gy}$ in order to cause severe skin dermatitis. It is well known, that repeated low doses of ionizing radiation causes chronic skin dermatitis. It is also known, that AP-1 transcription factor is activated by irradiation and that this activation causes induction of Tumor Growth Factor (TGF- $\beta$ ) in the skin and that this TGF- $\beta$ is partly responsible for radiation-induced fibrosis [23]. We are currently investigating the potential altered immune cell proportions in irradiation-induced skin dermatitis in mice.

Acute injury, which occurs within hours to weeks after radiation exposure, results from immediate structural tissue damage, generation of short-lived free radicals [24], irreversible double-stranded breaks in nuclear and mitochondrial DNA, and initiation of an inflammatory response in the epidermis and dermis [18-21]. Repeated exposure to low-dose ionizing radiation does not allow time for cells to repair DNA or tissue damage. The accumulation of radiation-induced changes to dermal vasculature, appendage structures, and epidermal stem cells results in progression of irradiation dermatitis through characteristic stages of severity.

Irradiation-induced dermatitis represents one of the most important dose limiting factors in radiotherapy [21,22]. In the present study we are focusing on the acute form of radiotherapy induced skin dermatitis, which normally develops within a few weeks after exposure to a high dose of ionizing radiation. The skin dermatitis shows erythema, epilating, dry- and moist-desquamation and erosion/ulceration. In current studies in mice we are looking into the occurrence of chronic dermatitis. In a recent study the outcome and toxicity of patients with anal cancer undergoing Intensity-modulated Radiation Therapy, IMRT, or Conformal Radiation Therapy (3D-CRT) was evaluated. The study demonstrated that IMRT could reduce severe acute skin toxicity significantly $(\mathrm{p}<0.001)$ compared to $3 \mathrm{D}-\mathrm{CRT}$. No toxicity grade 4 was observed and G2/3 toxicity was reduced from $94.6 \%$ to $63.2 \%$ ) [25]. No effect on overall survival, progression-free survival or on local control was observed. Irradiation given in a single high dose or in sub-erythemal doses for 14 days have been demonstrated to exert negative effects on the epidermal barrier, leading to barrier disruption [24]. In contrast, low doses of irradiation exert positive effects on the epidermal barrier, without clinically evident inflammation or barrier disruption. The aim of our study was to see whether a newly developed anti irradiation drug could prevent stage IV skin dermatitis. That is why we used irradiation with a single high dose of $30 \mathrm{~Gy}$ in order to demonstrate negative effects of irradiation on the epidermal barrier. In a recent study promising results were published with treatment of a Toll-like receptor 5 agonist. The agonist reduced irradiation induced skin dermatitis associated with epidermal hyperplasia and dermal inflammation through activation of endogenous antioxidants reducing free radicals [26].

Currently, no effective treatment exists to prevent or treat skin dermatitis in humans. Therefore, there is a huge unmet clinical need to develop new effective treatments of radiation induced skin dermatitis. The promising results with BP-C2 in the present study makes hope for future treatments of radiotherapy-induced skin dermatitis. Current studies will hopefully demonstrate the effect of BP-C2 on chronic skin dermatitis i.e., the manifestation of fibrosis-related irradiation-induced dermatitis and the potential genetic background. We are now waiting for the additional studies on the predictive ability of synergistically acting genes, both in tissues and in peripheral blood, which probably will increase our knowledge further on the mechanism behind acute as well as chronic skin dermatitis after irradiation.

Overall, many trials have been conducted to evaluate a large variety of products and methods in prevention of acute radiation-induced skin dermatitis. However, they do not support a general consensus on a superior product that should be used in this setting. Concerning treatments assessed for the management of radiation-induced skin reactions include topical steroid creams [27], non-steroidal creams $[17,28]$ and herbal remedies $[29,30]$. Bordelaya et al. have recently demonstrated that toll-like 5 agonist protects mice from dermatitis and oral mucositis caused by local radiation of head and neck cancer [26] 
The small number and large variety of trials make it difficult to draw any conclusions concerning the management of radiation induced skin dermatitis. Thus trials assessing products used for prevention of acute induced skin injuries as well as treatments for radiation-induced skin reactions, especially moist desquamation and ulceration reactions (grades III and IV), are needed.

\section{Conclusion}

In summary, the present study has shown that topical application of a novel Complex of Benzene-Poly-Carboxylic Acid and Molybdenum (BP-C2) protects against irradiation-induced dermatitis whether BP$\mathrm{C} 2$ is used prophylactically. Moreover, when skin ulcers have occurred after irradiation it can be cured completely after topical application of BP-C2. These results may indicate that BP-C2 can be used for prevention and treatment of skin injury following irradiation exposure.

\section{Acknowledgements}

We thank CEO Mr. Stig Løfberg, Meabco A/S, Copenhagen, Denmark for supporting the study with an unrestricted grant. Chief of Clinical operations Karen Møller, Meabco A/S is acknowledged for her language support.

\section{References}

1. Hickok JT, Morrow GR, Roscoe JA, Mustian K, Okunieff P (2005) Occurrence, severity and longitudinal course of twelve common symptoms in 1129 consecutive patients during radiotherapy for cancer. J Pain Symptom Manage 30: 433-442.

2. Hymes SR, Strom EA, Fife C (2006) Radiation dermatitis: clinical presentation, pathophysiology, and treatment 2006. J Am Acad Dermatol 54: 28-46.

3. Brown KR, Rzucidlo E (2011) Acute and chronic radiation injury. J Vasc Surg 53: $15 \mathrm{~S}-21 \mathrm{~S}$

4. McQuestion M (2011) Evidence-based skin care management in radiation therapy: clinical update. Semin Oncol Nurs 27: e1-17.

5. Salvo N, Barnes E, van Draanen J, Stacey E, Mitera G, et al. (2010) Prophylaxis and management of acute radiation-induced skin reactions: a systematic review of the literature. Curr Oncol 17: 94-112.

6. Ryan JL (2012) lonizing radiation: the good, the bad, and the ugly. J Invest Dermatol 132: 985-993.

7. Archambeau JO, Pezner R, Wasserman T (1995) Pathophysiology of irradiated skin and breast. Int J Radiat Oncol Biol Phys 31: 1171-1185.

8. Bernier J, Bonner J, Vermorken JB, Bensadoun RJ, Dummer R, et al (2008) Consensus guidelines for the management of radiation dermatitis and coexisting acne-like rash in patients receiving radiotherapy plus EGRF inhibitors for the treatment of squamous cell carcinoma of the head and neck. Ann Oncol 19: 142-149.

9. Hopewell JW (1990) The skin: its structure and response to ionizing radiation. Int J Radiat Biol 57: 751-773.

10. Cipollaro VA (2001) Radiation dermatitis today. J Eur Acad Dermatol Venereol 15: $300-301$

11. McQuestion M (2006) Evidence-based skin care management in radiation therapy. Semin Oncol Nurs 22: 163-173.

12. Gerlach MA (2005) Wound care issues in the patient with cancer. Nurs Clin North Am 40: 295-323.

13. Bykov V (2014) Report on the research study "Experimental Investigation of the Radioprotective Effect of BP-C2 in Mice and Rats" In Ministry of Defense of the Russian Federation. Main Directorate of Military Medicine. SM Kirov Military Medical Academy. UDC 613.648 (047.3) 1-48.

14. Fares F, Azzam N, Fares B, Larsen S, Lindkaer-Jensen S (2014) Benzenepoly-carboxylic acid complex, a novel anti-cancer agent induces apoptosis in human breast cancer cells. PLoS One 9: e85156.
15. Larsin S, Butthongkomvong K, Manikhas A, Trishkina E, Poddubuskaya E et al. (2014) BP-C1 in the treatment of patients with stage IV breast cancer: randomized, double-blind, placebo-controlled multicenter study and an additional open-label treatment phase. Br Can Targ Ther 6: 179-189.

16. Kumar S, Kolozsvary A, Kohl R, Lu M, Brown S, et al. (2008) Radiationinduced skin injury in the animal model of scleroderma: implications for postradiotherapy fibrosis. Radiat Oncol 3: 40.

17. Ertekin MV, Tekin SB, Erdogan F, Karslioglu I, Gepdiremen A, et al. (2004) The effect of zinc sulphate in the prevention of radiation-induced dermatitis. $J$ Radiat Res 45: 543-548.

18. Elting LS, Keefe DM, Sonis ST, Garden AS, Spijkervet FK, et al. (2008) Patientreported measurements of oral mucositis in head and neck cancer patients treated with radiotherapy with or without chemotherapy: demonstration of increased frequency, severity, resistance to palliation, and impact on quality of life. Cancer 113: 2704-2713.

19. Larsen S. Fares F. Lindkær-Jensen S (2014) Investigations into the antiradition effects of BP-C2, a benzene-poly-carboxylic acid complex with molybdenum. J Clin Oncol 32: 5s.

20. Lindkær-Jensen S, Larsen S, Habib N, Lindkær-Jensen N, Fagertun H (2015) Positive effects on hematological and biochemical imbalances in patients with metastatic breast cancer stage IV, of BP-C, a new anticancer substance. Drug Des Devel Ther 9: 1481-1490.

21. Haratake A, Uchida Y, Schmuth M, Tanno O, Yasuda R, et al (1997) UVBinduced alterations in permeability barrier function: roles for epidermal hyper proliferation and thymocyte-mediated response. J Invest Dermatol 108: 769-775.

22. Takagi Y, Nakagawa H, Kondo H, Takema Y, Imokawa G (2004) Decreased levels of covalently bound ceramide are associated with ultraviolet B-induced perturbation of the skin barrier. J Invest Dermatol 123: 1102-1109.

23. Martin M, Vozenin MC, Gault N, Crechet F, Pfarr CM, et al. (1997) Coactivation of AP-1 activity and TGF-beta1 gene expression in the stress response of normal skin cells to ionizing radiation. Oncogene 15: 981-989.

24. Permatasari F, Zhou B, Luo D (2013) Epidermal barrier: Adverse and beneficia changes induced by ultraviolet $B$ irradiation depending on the exposure dose and time (Review). Exp Ther Med 6: 287-292.

25. Koerber SA, Slynko A, Haefner MF, Krug D, Schoneweg C, et al. (2014) Efficacy and toxicity of chemoradiation in patients with anal cancer--a retrospective analysis. Radiat Oncol 9: 113.

26. Burdelya LG, Gleiberman AS, Toshkov I, Aygun-Sunar S, Bapardekar M, et al. (2012) Toll-like receptor 5 agonist protects mice from dermatitis and oral mucositis caused by local radiation: implications for head-and-neck cancer radiotherapy. Int J Radiat Oncol Biol Phys 83: 228-234.

27. Bostrom A, Lindman H, Swartling C, Berne B, Bergh J (2001) Poten corticosteroid cream (mometasone furoate) significantly reduces acute radiation dermatitis: results from a double-blind, randomized study. Radiother Oncol 59: 257-265.

28. Murakami R, Baba Y, Nishimura R, Furusawa M, Yokoyama T, et al. (1997) The effect of azelastine on acute radiation dermatitis in mice models. Int Radiat Oncol Biol Phys 37: 907-911.

29. Wang XJ, Lin S, Kang HF, Dai ZJ, Bai MH, et al. (2013) The effect of RHIZOMA COPTIDIS and COPTIS CHINENSIS aqueous extract on radiation-induced skin injury in a rat model. BMC Complement Altern Med 13: 105.

30. Jagetia GC, Rajanikant GK (2005) Curcumin treatment enhances the repair and regeneration of wounds in mice exposed to hemibody gamma-irradiation Plast Reconstr Surg 115: 515-528. 